\title{
Measurement of Lung Density in Congestive Heart Failure by Computed Tomography
}

\author{
Masanori Nomura, M.D., Yutaka Mryagr, M.D., Keiji TaGhi, M.D., \\ Yoshiyuki Sakabe, M.D., Yasuhiko SakaI, M.D., Hitoshi Hishida, M.D., \\ Yasushi Mizuno, M.D., Fumio Sasaki, M.D.,* \\ and Sukehiko KogA, M.D.*
}

\section{Summary}

The computed tomography (CT) number within the region of interest (ROI) was used as a parameter to assess lung density in patients with congestive heart failure. Thirty-eight patients with valvular heart disease (VHD) and 34 patients with ischemic heart disease (IHD) were studied. Based on the New York Heart Association (NYHA) classification, 24 VHD patients were in class I or II (VHD I-II) and the other 14 were in NYHA class III or IV (VHD III-IV). Eighteen patients with IHD were in NYHA class I or II (IHD I-II) and 16 were in class III or IV (IHD III-IV). The CT number was measured bilaterally at the upper, middle and lower levels of the chest and compared with the corresponding values in 21 normal subjects (Group N). In a preliminary study on Group $\mathrm{N}$, the CT numbers were insensitive to the size of the ROI, but were closely related to its location.

In clinical applications, the mean values of the $\mathrm{CT}$ numbers in all six lung fields increased in the order of IHD I-II, to VHD I-II, IHD III-IV and VHD III-IV. Except for patients in IHD I-II, they were significantly larger than in Group N. The relationship between the CT number and the systolic and mean pulmonary arterial pressures and the pulmonary capillary wedge pressure were evaluated in 36 patients. Significant correlations were obtained in all six lung fields $(r=0.65-0.78, p<0.001)$. The results suggest that measurement of lung density by CT is useful for the quantitative evaluation of the severity of disease in patients with congestive heart failure.

From the Department of Internal Medicine, Department of Radiology,* Fujita-Gakuen Health University, School of Medicine, Toyoake, Aichi, Japan.

Address for reprint: Masanori Nomura, M.D., Department of Internal Medicine, Fujita-Gakuen Health University, School of Medicine, 1-98 Kutsukake-cho, Dengakugakubo, Toyoake, Aichi 470-11, Japan.

Received for publication December 26, 1983. 


\section{Additional Indexing Words:}

\section{CT number Pulmonary artery pressure Pulmonary edema}

7 HERE are only a few non-invasive, quantitative methods for the evalua1 tion of the severity of pulmonary congestion. Since chest roentgenography is a simple method, many reports have been published on the relationship between chest roentgenographic findings, clinical signs and indices of hemodynamics. ${ }^{1)-10}$ ) Recently, measurements of lung fluid volume have been made possible by the use of gamma ray compton backscatter ${ }^{11)}$ or radioisotopes ${ }^{12), 13)}$ in the presence of pulmonary congestion. More recently, the evaluation of lung density by computed tomography (CT) has been reported. ${ }^{15)-21)}$ With the application of CT, it now appears possible to evaluate the changes of fluid content in pulmonary congestion in a more quantitative manner by detecting minute differences in the attenuation of roentgenograms. In the present study, chest CT pictures were obtained in patients with congestive heart failure due to different causes, and the CT numbers in the lung fields of the patients were compared with those in healthy subjects. In patients in whom the pulmonary arterial pressure and pulmonary capillary wedge pressure were measured, the relationships between the GT numbers of the lung fields and the pressures were assessed.

\section{Subjects ANd Methods}

\section{Subjects}

Of the 46 men and 26 women studied, 38 (22 to 70 years of age) had valvular heart diseases (VHD) and 34 (30 to 79 years of age) had ischemic heart diseases (IHD). All patients were further divided into 2 groups ac-

Table I. Characteristics of Subjects

\begin{tabular}{|c|c|c|c|}
\hline & $\begin{array}{l}\text { Number } \\
\text { (female) }\end{array}$ & $\begin{array}{l}\text { Age (mean) } \\
\text { (years) }\end{array}$ & Primary disease and number \\
\hline VHD I-II & $24(11)$ & $24-61(44.1)$ & $\begin{array}{l}\text { MS 6, MR 5, AR 2, AS 3, ARS 3, MS+ } \\
\text { AR 3, MS+ARS 1, MRS +AR } 1\end{array}$ \\
\hline VHD III-IV & $14(6)$ & $22-70(50.1)$ & $\begin{array}{l}\text { MS 4, MR 1, MRS 2, AR 1, ARS } 2, \mathrm{MS}+ \\
\text { ARS 2, MR + AR 1, MR+ARS } 1\end{array}$ \\
\hline IHD I-II & $18(2)$ & $30-70(54.7)$ & MI 13, AP 5 \\
\hline IHD III-IV & $16(6)$ & $54-79(67.5)$ & MI 8, others 8 \\
\hline Normal & $21(1)$ & $22-54(32.7)$ & \\
\hline
\end{tabular}

Roman numerals indicate NYHA class. Abbreviations: VHD=valvular heart disease ; IHD= ischemic heart disease; $M S=$ mitral stenosis; $M R=$ mitral regurgitation; $M R S=M S+M R$; $\mathbf{A R}=$ aortic regurgitation ; $A S=$ aortic stenosis; $A R S=A R+A S ; M I=$ acute myocardial infarction; $\mathbf{A P}=$ angina pectoris. 
cording to the functional state based on the New York Heart Association (NYHA) classification (Class I, II, III or IV), as shown in Table I. For comparison, 21 healthy normal subjects, aged 22 to 53 years, without any cardiovascular or respiratory disease were also studied (Group N). The relationships between the CT numbers and pulmonary arterial and pulmonary capillary wedge pressures were evaluated in 12 patients with VHD I-II, 10 with VHD III-IV, 6 with IHD I-II and 8 with IHD III-IV.

\section{Methods}

CT examination was performed using a Hitachi CT-W3 with the subjects in the supine position. The computed radiographic picture (GR) of the chest in the ventro-dorsal direction was taken at a tube voltage of $120 \mathrm{KV}$ and tube current of $200 \mathrm{~mA}$ while the patient held his breath at full inspiration. Using $\mathrm{CR}$ as a reference, pictures were obtained at three levels of the chest (aortic arch, hilar portion and the left 4th arch of a cardiac silhouette), and

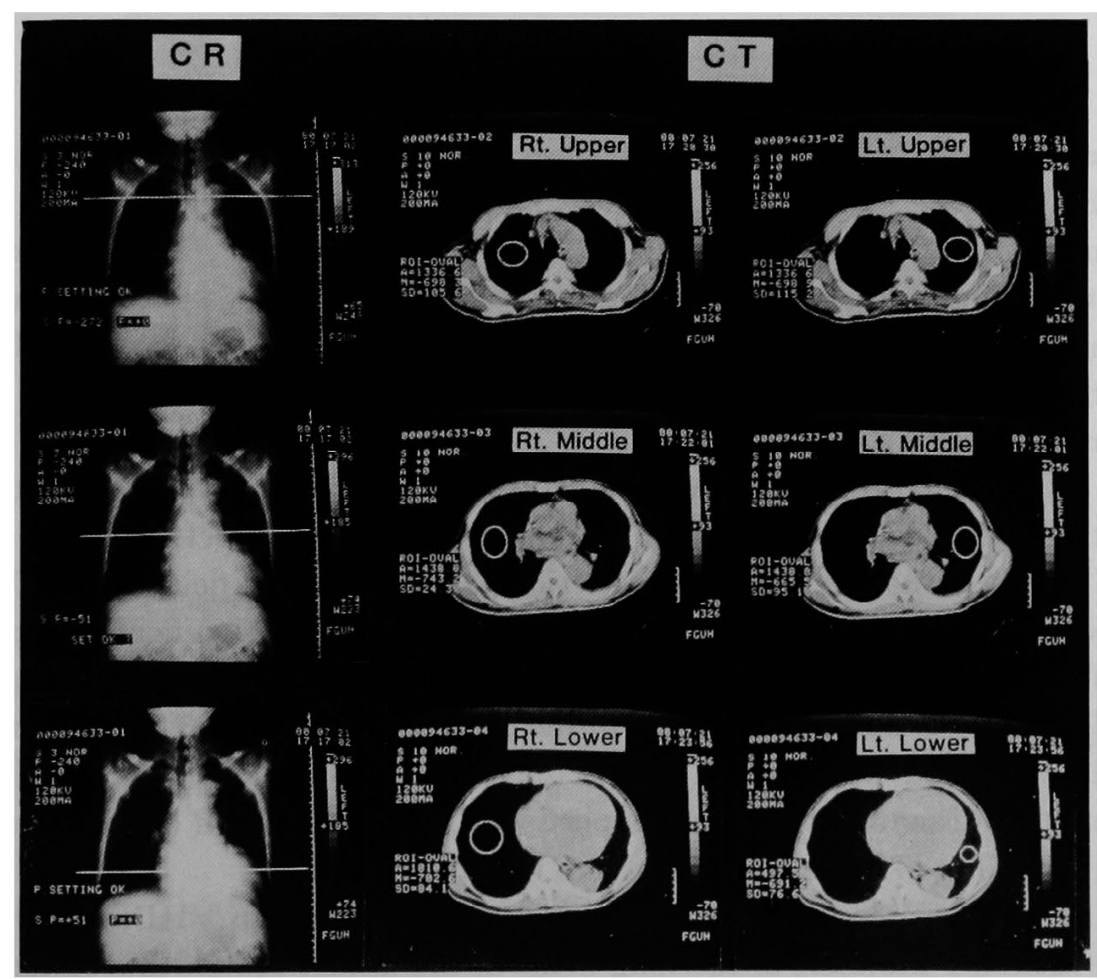

Fig. 1. The mean value of the CT number measured within the region of interest (ROI), positioned at the center of the outer two-thirds of each lung field at the level of the aortic arch (upper), hilar portion (middle) and the left fourth arch of a cardiac silhouette (lower). 
stored on magnetic tapes. These three levels represent the upper, middle and lower chest, respectively. The region of interest (ROI) of arbitrary size was set at the center of the outer two-thirds of the cross section of right and left lung fields at each level to exclude major hilar vessels. The area of pleural effusion was also avoided. The mean value of the GT numbers within the ROI was used for statistical analysis (Haunsfield Unit, HÜ) (Fig. 1). The relationship between pulmonary arterial and pulmonary capillary wedge pressures and the CT numbers of the lung fields were evaluated in 36 patients in whom CT were taken while a Swan-Ganz catheter was in place or within 24 hours prior to diagnostic right heart catheterization. These pressures were measured during quiet respiration by connecting the catheter to a transducer (Siemens 746 or Statham P23 Db). Analysis of variance was used for the assessment of the significance of difference between the groups, and paired $t$-test was used for the statistical analysis in the normal subjects.

\section{RESUlts}

1. Influence of the size and site of the ROI on the CT number

The size of the ROI was increased stepwise from about 150 to 2,500 pixels at the middle level of the right side of the chest and the CT number within this area was measured in 21 healthy subjects (Fig. 2). The mean value of the CT number varied from -822 to $-824 \mathrm{HU}$ (Table II), indicating that it is insensitive to changes in the size of the ROI. However, the CT number tended to become smaller as the size of the ROI increased. Therefore, in the present study, the size of the ROI was set at approximately 1,500 pixels.

The influence of the site of the ROI on the CT number was then studied at the middle level of the chest in the same normal subjects (Fig. 3). The CT number was largest in the posterior part of the lung field bilaterally followed in order by the central and anterior parts. A significant difference was noted in the CT numbers between the posterior part and both the anterior and central part on the right side of the chest, as well as between the posterior and anterior parts on the left side of the chest (Table III, Fig. 4). The ROI was set consistently in the central portion of the lung field in order to exclude the possibility of differences related to its location.

2. Measurement of the CT number in patients with VHD and IHD

The CT numbers of the lung fields at the upper, middle and lower level of the chest were compared between the 4 patient groups and normal group (Table IV). The number increased at all levels in the order: Group N, IHD I-II, VHD I-II, IHD III-IV and VHD III-IV (Fig. 5). In IHD 


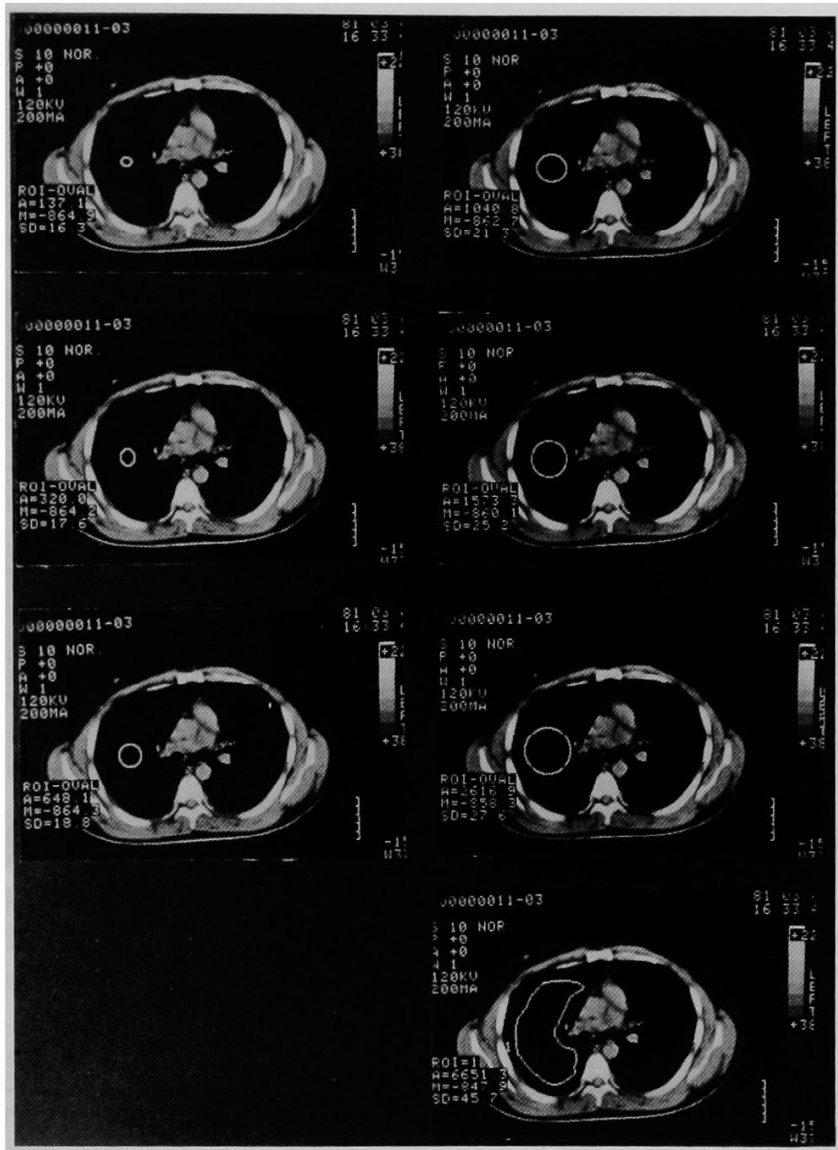

Fig. 2. Influence of the size of the ROI on the CT number in normal subjects. The size of the ROI was increased stepwise. In the bottom picture, the ROI comprised the entire lung field, with care taken to avoid sampling hilar great vessels.

Table II. Influence of the Size of the ROI on the CT Number in Normal Subjects

\begin{tabular}{l|rrrrrrr}
\hline $\begin{array}{c}\text { Area of ROI } \\
\text { (pixels) }\end{array}$ & 150 & 300 & 600 & 1,000 & 1,500 & 2,500 & 6,500 \\
\hline CT number & -824 & -824 & -824 & -824 & -822 & -822 & -815 \\
SD & 40 & 32 & 29 & 26 & 25 & 24 & 23
\end{tabular}

I-II, the CT numbers were not very different from those in Group $\mathbf{N}$ at any level of the chest. On the other hand, CT numbers in VHD I-II, IHD III-IV and VHD III-IV were larger than those in Group N at all levels of the chest. Analysis of variance revealed significant difference $(p<0.01)$ between the groups at each level of the lungs. Compared to values at the 


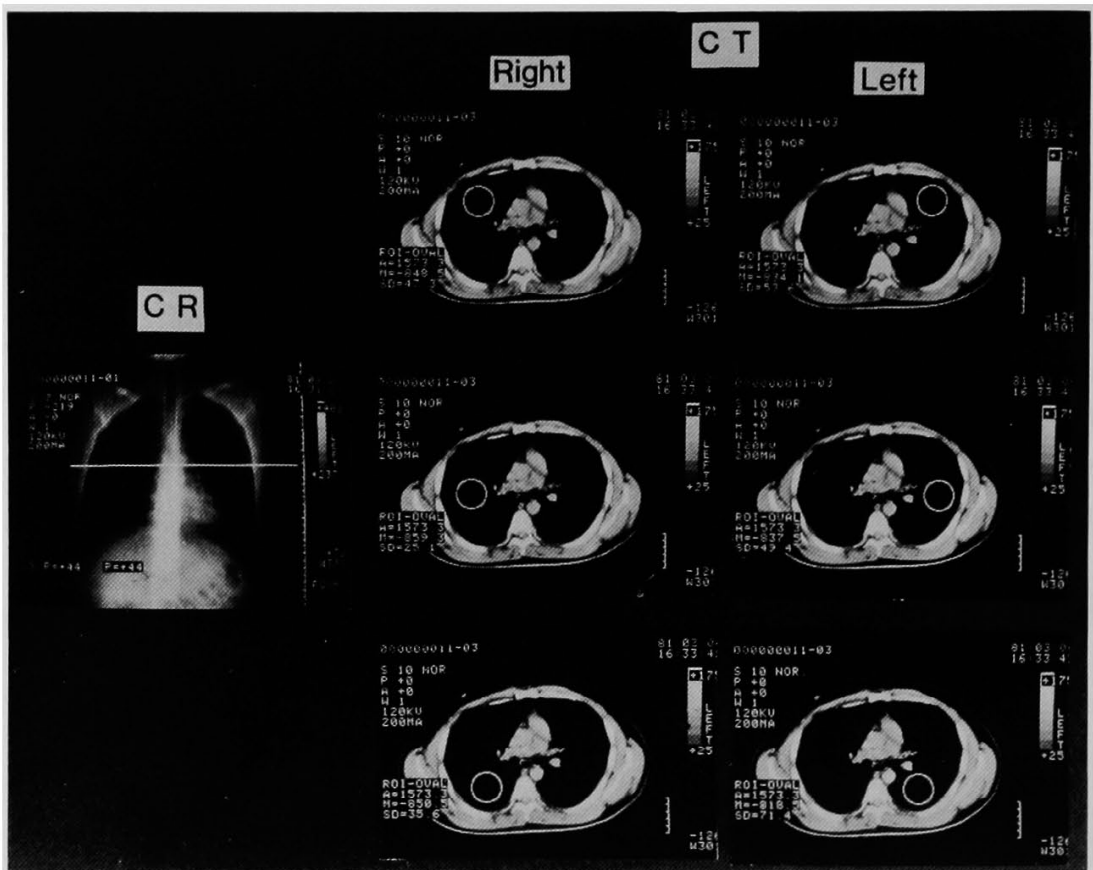

Fig. 3. Influence of the site of the ROI on the CT number in normal subjects. At the middle level lung field, the ROI was placed at the anterior, central and posterior part of the lungs.

Table III. Influence of the Site of the ROI on the CT Number in Normal Subjects

\begin{tabular}{l|c|c}
\hline & Right & Left \\
\hline Anterior & $-827 \pm 23$ & $-821 \pm 23$ \\
Central & $-821 \pm 24$ & $-809 \pm 25$ \\
Posterior & $-800 \pm 30$ & $-791 \pm 32$
\end{tabular}

upper and lower levels of the bilateral lung fields, those at the middle level were the smallest in Group N, IHD I-II and VHD I-II. They were largest in the left lung in IHD III-IV and VHD III-IV (Fig. 5).

3. Relationship between the CT number and pulmonary arterial and pulmonary capillary wedge pressures

Significant positive correlations were noted between pulmonary arterial pressure or pulmonary capillary wedge pressure and the CT number at the upper, middle and lower bilateral lung fields of the chest (Table V). On the left side of the chest, the highest correlation was noted at the lower level, followed by the middle and upper levels. On the right side, a high correlation was found at the middle level (Figs. 6-8). 
CT

Number

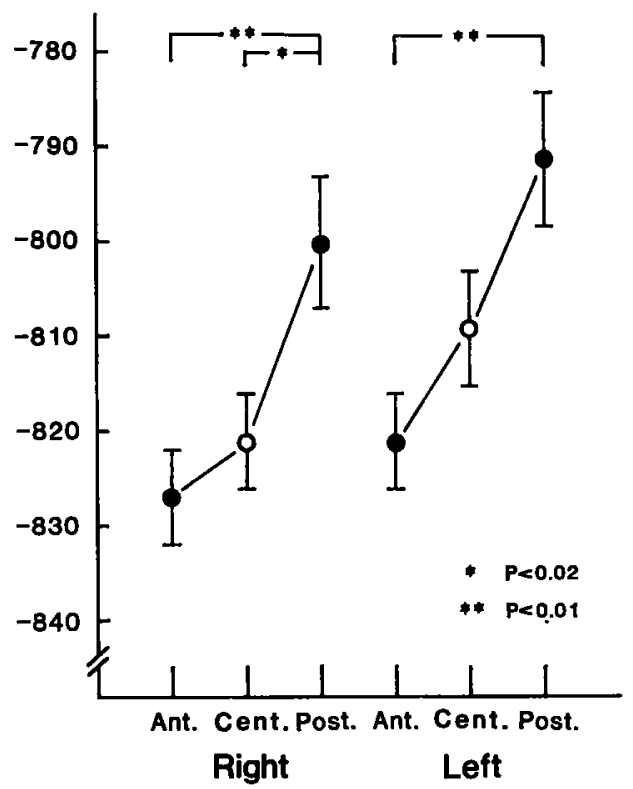

Fig. 4. Changes of the CT number at various sites (anterior, central, posterior) of the lung in 21 normal subjects. Open circles and bars represent the mean values and SEs measured at the middle part of the lung.

Table IV. Values of CT Number Measured at Each Level of the Lungs

\begin{tabular}{l|ccc|ccc}
\hline & \multicolumn{4}{|c|}{ Right } & \multicolumn{3}{c}{ Left } \\
\cline { 2 - 6 } \cline { 5 - 7 } & Upper & Middle & Lower & Upper & Middle & Lower \\
\hline VHD I-II $(n=24)$ & $-759 \pm 60$ & $-780 \pm 50$ & $-743 \pm 58$ & $-759 \pm 59$ & $-770 \pm 56$ & $-751 \pm 70$ \\
VHD III-IV $(n=14)$ & $-679 \pm 116$ & $-701 \pm 76$ & $-678 \pm 80$ & $-686 \pm 80$ & $-653 \pm 99$ & $-664 \pm 110$ \\
IHD I-II ( $=18)$ & $-789 \pm 49$ & $-815 \pm 38$ & $-775 \pm 64$ & $-800 \pm 34$ & $-810 \pm 46$ & $-787 \pm 52$ \\
IHD III-IV ( $n=16)$ & $-727 \pm 79$ & $-736 \pm 64$ & $-722 \pm 70$ & $-725 \pm 71$ & $-689 \pm 90$ & $-700 \pm 72$ \\
Normal $(n=21)$ & $-792 \pm 26$ & $-821 \pm 24$ & $-773 \pm 40$ & $-792 \pm 31$ & $-809 \pm 25$ & $-785 \pm 30$
\end{tabular}

Values represent mean and standard deviation.

\section{Discussion}

Many methods have been used to assess the severity of pulmonary congestion in patients with congestive heart failure. Dyspnea as a subjective symptom is useful for the approximate estimation of the severity of pulmonary congestion. Killip's classification ${ }^{7,14)}$ also suggests a relationship between the pulmonary auscultatory findings and cardiac failure.

A routine chest roentgenogram is most frequently used clinically and its 


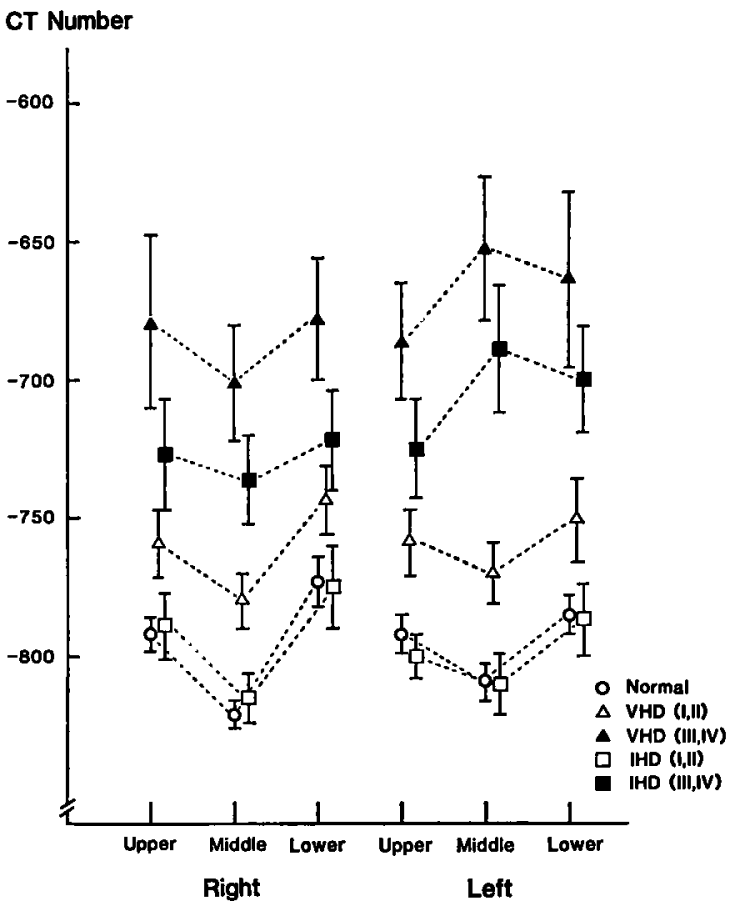

Fig. 5. The CT number measured at the three levels (upper, middle, lower) of the lungs. Open and solid triangles and bars represent mean values and SEs in VHD I-II and VHD III-IV. Open and solid boxes and bars represent mean values and SEs in IHD I-II and IHD III-IV. Open circles and bars represent mean values and SEs in normal subjects. Analysis of variance revealed significant difference $(p<0.01)$ between the groups at each level of the lungs.

Table V. Correlation Coefficient between Pressure Indices and the CT Number in Each Lung Field

\begin{tabular}{l|ccc|ccc}
\hline & \multicolumn{3}{|c|}{ Right } & \multicolumn{3}{c}{ Left } \\
\cline { 2 - 7 } & Upper & Middle & Lower & Upper & Middle & Lower \\
\hline PAP (syst.) & 0.63 & 0.67 & 0.65 & 0.68 & 0.72 & 0.77 \\
PAP (mean) & 0.71 & 0.72 & 0.69 & 0.74 & 0.77 & 0.78 \\
PCWP & 0.65 & 0.69 & 0.65 & 0.70 & 0.70 & 0.71
\end{tabular}

All values are statistically significant $(\mathrm{p}<0.001)$.

diagnostic value is high. Even in the early stages of heart failure, with a paucity of physical findings, it is possible to suspect abnormalities of pulmonary circulatory dynamics from the chest roentgenogram.. ${ }^{2), 3)}$ However, the changes in density which depend upon the conditions of radiographic interpretation are apt to be influenced by a physician's subjective considerations. 


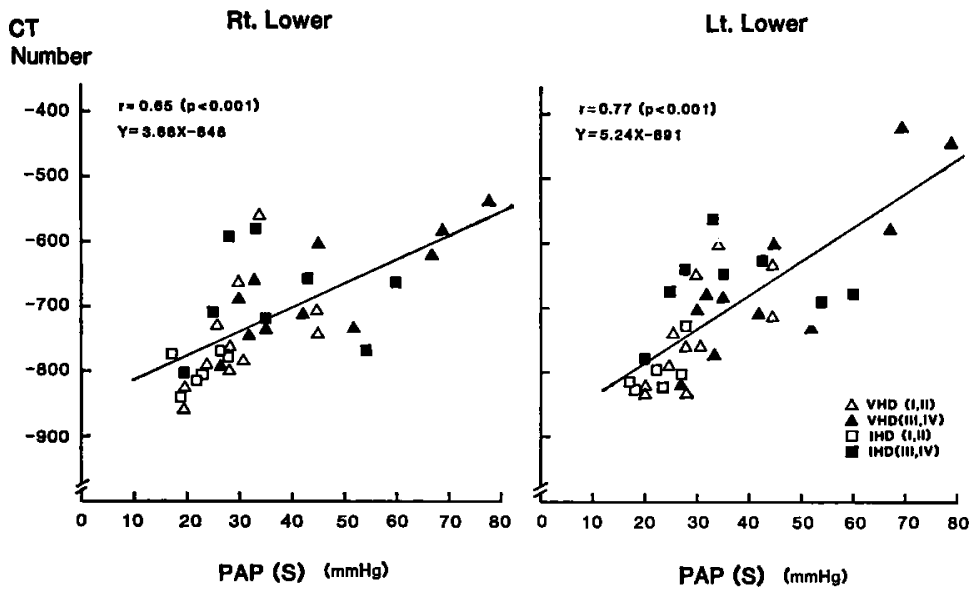

Fig. 6. Correlation between systolic pulmonary arterial pressure [PAP (S)] and CT number in the right and left lower lung fields. Symbols are same as in Fig. 5.

CT
Rt. Lower
Lt. Lower
Number
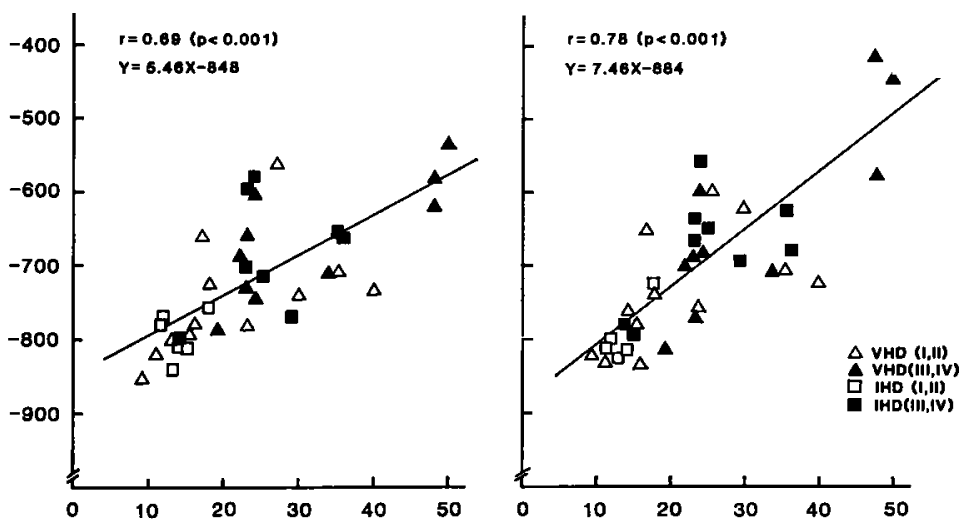

PAP (M) (mmHg)

Fig. 7. Correlation between mean pulmonary arterial pressure [PAP (M)] and CT number in the right and left lower lung fields. Symbols are same as in Fig. 5.

The measurement of lung density by the use of gamma ray compton backscatter or methods for the estimation of lung fluid volume have been introduced. ${ }^{11)-13)}$ The use of cardiac catheterization permits quantitative evaluation by the measurement of intracardiac pressures. In the diagnosis and evaluation of the severity of congestive heart failure, non-invasive methods which are safe, accurate and highly reproducible are desirable. In order to 


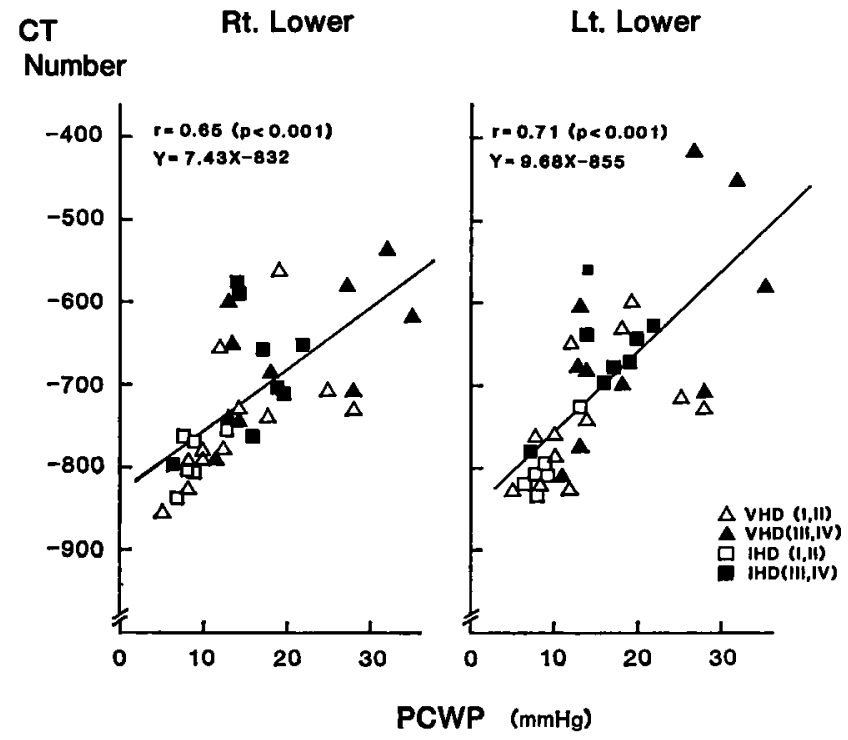

Fig. 8. Correlation between pulmonary wedge pressure (PCWP) and CT number in the right and left lower lung fields. Symbols are same as in Fig. 5.

meet such demands, attention was focused on the CT method, which has become increasing useful in the diagnosis of diseases of the chest. CT has been used previously in studies of lung density. ${ }^{15)-18)}$ It has been used to detect abnormalities more readily than other radiological methods in diseases which may decrease lung density, such as pulmonary emphysema, or increase fluid accumulations, such as pulmonary congestion. ${ }^{15), 16), 21)}$ The measurement of lung density by the CT method is little influenced by the thorax, rib and soft tissue, and reflects the changes of fluid content in the thorax. ${ }^{16), 20)}$ In the evaluation of cardiopulmonary function, the influence of respiration on these factors cannot be ignored. ${ }^{16)}$ Lung densities at repeated maximum inspiration showed high reproducibility. With regard to the influence of body position, the density of the anterior and posterior portions of the lung reverses by changing from supine to prone position. ${ }^{15), 20), 21)}$ This is explained by the differences in blood flow due to gravity. ${ }^{17}$ Taking these factors into consideration, we performed the CT examinations in the supine position at maximum inspiration. In a preliminary study, the influence of the size and site of the ROI on the CT number was studied in healthy subjects. The CT number within the ROI was insensitive to changes in the size of the ROI. Even when the ROI was set to the entire lung field, the CT number did not differ significantly from the average ROI in this study (1,500 pixels). The cross-sectional view of the lung is not as homogeneous as water, and the density value varies due to heterogeneous constituents such as the blood vessels, 
interstitium and air. Thus, it was evident that the site of the ROI influenced the density value markedly. The peripheral lung (parenchyma) is thought to be homogeneous. For this reason, many investigators evaluated lung density by setting the ROI in the peripheral portion of the lung field. ${ }^{15), 19)-21)}$ The comparisons of CT numbers from the bilateral lung fields revealed that CT numbers from the posterior part are significantly larger than those from the anterior part. The difference probably reflects altered blood flow due to gravity, as was pointed out by Kreel et al. ${ }^{17)}$ In the present study, therefore, the ROI was set at the center of the lung field in the anteroposterior direction. In some patients with marked cardiomegaly, though, the ROI in the left lower lung field had to be shifted somewhat posteriorly. Hardly any differences in the CT numbers were noted at the upper, middle and lower levels of the bilateral lung fields at a total of 6 sites between Group N and IHD I-II. IHD I-II consisted of patients who, more than 2 weeks after the onset of acute myocardial infarction, had a favorable course without complications and those with angina pectoris actually belong to NYHA class I. None of these patients showed clinical signs of heart failure. The mean ages in Group N and IHD I-II were 33 and 55 years, respectively. Although they were significantly different, no difference in CT numbers was observed between these 2 groups. Rosenblum found no influence of aging on lung density, except for children. ${ }^{15), 20)}$

VHD I-II consist of patients with mild or no clinical signs of heart failure. The GT numbers in this group were significantly larger than those in the former 2 groups at each level. In IHD III-IV, the CT numbers were larger than those in the above 3 groups. In VHD III-IV, they were the largest of all the groups studied in every level of the lung fields.

Clinically, there is a close relationship between the NYHA classification and pulmonary congestion. Development of pulmonary congestion indicates an increase in fluid content in the lung, which will be reflected in an increase in lung density measured by CT (as seen in those with VHD III-IV). Rosenblum et al reported lung densities at the lung base, carina and apex of -739 $\mathrm{HU},-748 \mathrm{HU}$ and $-740 \mathrm{HU}$, respectively, in normal subjects. ${ }^{20)}$ A similar relation was seen in our study in normal subjects or in patients without clinical signs of heart failure or with mild heart failure. However, in IHD III-IV and VHD III-IV the CT numbers of the left lung at the middle level were larger than those in the upper and lower levels of the same left lung. Although this phenomenon was absent in the right lung in these groups, the differences in the CT numbers between the middle and lower level lung fields of the right lung were small in comparison with other groups. This suggests that changes in distribution of vascular flow pattern in the lungs accompanied 
the development of heart failure.

The correlation between pulmonary arterial and pulmonary capillary wedge pressures and the CT number in each lung field was then studied. Systolic and mean pulmonary arterial pressures and pulmonary capillary wedge pressure showed a significant correlation with CT numbers in each lung field. The mean pulmonary arterial pressure showed a higher correlation coefficient than the other pressure indices. The CT number in the lower field of the left lung and the middle field of the right lung showed the highest correlation with each pressure value. Numerous attempts have been made to obtain information on intracardiac pressure noninvasively. ${ }^{5)-8 \text { ) }}$ $\mathrm{McHugh}$ et al classified the degree of severity of left heart failure in patients with acute myocardial infarction on roentgenographic film into 4 grades in order to study the correlation with pulmonary wedge pressure. A good correlation was noted between grades in the chest roentgenographic picture and pulmonary wedge pressure. ${ }^{5)}$ Kostuk et al also studied the relationship between chest roentgenographic findings and hemodynamic changes in acute myocardial infarction. ${ }^{8)}$ Turner et al suggested the possibility of estimating the pulmonary venous and arterial pressure from the chest roentgenogram. ${ }^{6)}$ Grainger et al pointed out the absence of the septal line in the chest X-ray when the pulmonary wedge pressure is less than $18 \mathrm{mmHg}$ and the diastolic pulmonary arterial pressure less than $25 \mathrm{mmHg} .{ }^{11}$ Wolk et al, on the other hand, suggested a poor relationship between these findings. ${ }^{7}$ Thus, the relationship between findings of pulmonary congestion in chest roentgenogram and pulmonary arterial pressure, especially pulmonary wedge pressure, is not always constant. However, $\mathrm{McHugh}$ et $\mathrm{al}^{(5)}$ and Kostuk et al ${ }^{8)}$ demonstrated the presence of time delay between the chest roentgenographic findings and pressure indices, which are thought to be one of the major causes for lack of correlation. It is expected that these affect the correlation between intracardiac pressure and lung density. As mentioned above, significant correlations between extravascular blood volume of the lung and the mean or diastolic pulmonary arterial pressure and wedge pressure have been reported using double radioisotope indicator techniques. ${ }^{12), 13)}$ In our attempt to estimate the pulmonary arterial or pulmonary capillary wedge pressure using CT, good correlations were found between the CT numbers and these pressures, which indicates that GT numbers probably reflect fluid content of the lung in congestive heart failure. Pulmonary arterial or capillary wedge pressure are usually measured by the use of a Swan-Ganz catheter and are often used as reliable indicators of the severity of pulmonary congestion. In the state of pulmonary congestion, the pulmonary fluid volume increases. The CT method detects the changes in pulmonary fluid volume quantitatively 
as CT numbers which closely correlate with the pulmonary arterial and capillary wedge pressures. Although some limitation exists with the routine use of CT, this method is a simple quantitative method which should be especially useful in following patients with pulmonary congestion.

\section{REFERENCES}

1. Grainger RG: Interstitial edema and its radiological diagnosis. A sign of pulmonary venous and capillary hypertension. Br J Radiol 31: 201, 1958

2. Simon $\mathrm{M}$ : The pulmonary vessels in incipient left ventricular decompensation. Radiologic observations. Circulation 24: 185, 1961

3. Logue RB, Rogers JV, Gay BB: Subtle roentgenographic signs of left heart failure. Am Heart J 65: 464, 1963

4. Lassers BW, George M, Abserton JL, Geggins BR, Philip T: Left ventricular failure in acute myocardial infarction. Am J Cardiol 25: 511, 1970

5. McHugh TJ, Forrester JS, Adler L, Zion D: Pulmonary vascular congestion in acute myocardial infarction. Hemodynamic and radiologic correlations. Ann Int Med 76: 29, 1972

6. Turner AF, Lau FYK, Jacombson G: A method for the estimation of pulmonary venous and arterial pressures from the routine chest roentgenogram. Am J Roentgen 116: 97, 1972

7. Wolk MJ, Scheidt S, Killip T: Heart failure complicating acute myocardial infarction. Circulation 45: 1125,1972

8. Kostuk W, Barr JW, Simon AL, Ross J Jr: Correlations between the chest film and hemodynamics in acute myocardial infarction. Circulation 48: 624, 1973

9. Forrester JS, Diamond G, Chatterjee K, Swan HJC: Medical therapy of acute myocardial infarction by application of hemodynamic subsets (first of two parts). New Engl J Med 295: 1356,1976

10. Heitzman ER, Zitter FM: Acute interstitial pulmonary edema. Am J Roent Radium Ther Nucl Med 98: 291, 1976

11. Garnett ES, Webber CE, Coates G, Cockshott WP, Nahmias C, Lassen N: Lung density. Clinical method for quantitation of pulmonary congestion and edema. CMA Journal 116: 153, 1977

12. Luepker R, Liander B, Korscren M, Varnauskas E: Pulmonary extravascular and intravascular fluid volumes in resting patients. Am J Cardiol 28: 295, 1971

13. Biddle TL, Khanna PK, Yu PN, Hodges M, Shah PM: Lung water in patients with acute myocardial infarction. Circulation 49: 115, 1974

14. Killip $\mathrm{T}$, Kimball $\mathrm{T}$ : Treatment of myocardial infarction in a coronary care unit. A two year experience with 250 patients. Am J Cardiol 20: 457, 1967

15. Rosenblum LJ, Mauceri RA, Wellenstein DE, Vassano DA, Cohen WN, Heitzman ER: Computed tomography of the lung. Radiology 129: 521, 1978

16. Wegener $\mathrm{OH}$, Kieppe $\mathrm{P}$, Oeser $\mathrm{H}$ : Measurement of lung density by computed tomography. J Comput Assist Tomo 2: 263, 1978

17. Kreel L: Computed tomography of the thorax. in Computed Tomography 1977, ed by Norman D, Dorobdin M, Newton TH, Mosby, St Louis, p 49, 1977

18. Grossman ZD, Thomas FD, Gangne GRT, Mauceri RA, Cohen WN, Heitzman ER, Singh A: Transmission computed tomographic diagnosis of experimentally produced acute pulmonary vascular occlusion in the dog. Radiology 131: 767, 1979

19. Robinson PJ, Kreel L: Pulmonary tissue attenuation with computed tomography. Comparison of inspiration and expiration scans. J Comput Assist Tomo 3: 740, 1979

20. Rosenblum JF, Mauceri RA, Wellenstein DE, Thomas FD, Bassano DA, Raasch BN, Chamberlain CC, Heitzman ER: Density patterns in the normal lung as determined by computed 
tomography, Radiology 137: 409, 1980

21. Harada J, Kaneko K, Kawakami K, Tada S, Arai T: Lung density in mitral valve disease. Kokyu to Junkan $28: 501,1980$ (in Japanese) 